\title{
Minocycline Might Be an Adjunctive Therapy Option for the Treatment of COVID-19: In Silico Screening, Structure-affinity Relationship, and Literature Review
}

Bin Xiao ( $\sim$ michael-bin@163.com )

Laboratory of Clinical Pharmacy, Ordos Central Hospital, Ordos School of Clinical Medicine, Inner Mongolia Medical University

\section{Research article}

Keywords: SARS-CoV-2, old drugs, Mpro, molecular docking, minocycline

Posted Date: July 7th, 2020

DOI: https://doi.org/10.21203/rs.3.rs-40141/v1

License: (c) (1) This work is licensed under a Creative Commons Attribution 4.0 International License.

Read Full License 


\section{Abstract}

Up to now, there is no specific therapy for the globally ongoing COVID-19. To explore potential inhibitors of Severe Acute Respiratory Syndrome-Coronavirus-2 (SARS-CoV-2) for the treatment of novel coronavirus disease (COVID-19), in silico screening of 135 clinical drugs was performed targeting on 3chymotrypsin-like protease (3CLpro, or M pro ). Six drugs including anti-HIV drug (raltegravir), antibacterial drugs (cefonicid, cefoperazone, minocycline), and antidiabetic drugs (canaglifozin, glyburide) showed high binding affinities $(\leq-8.5 \mathrm{kcal} / \mathrm{mol}$ ) and interesting binding conformations compared with the designed co-crystal ligand N3 $(-7.7 \mathrm{kcal} / \mathrm{mol})$. In which the antibiotic minocycline, an inhibitor of bacterial ribosomal rRNA, showed the highest binding affinity $(-9.6 \mathrm{kcal} / \mathrm{mol})$. Valuable hydrogen bonding and hydrophobic interactions were found between minocycline and $\mathrm{M}^{\text {pro }}$ active site. Beside the hydrogen bond with Cys145, minocycline formed a Pi-Cation with His41, which strongly supported minocycline as a Michael Addition acceptor to bind with the catalytic site of $\mathrm{M}^{\text {pro }}$. The structure-affinity relationship was studied based on molecular docking of minocycline analogues. Literature review found that minocycline had both in vitro and in vivo broad-spectrum antiviral as well as anti-inflammatory activities, and the levels of a broad-spectrum of biological markers during minocycline administration were opposed to those of COVID-19 condition (both severe and non-severe). Minocycline deserves in vitro and in vivo evaluations as SARS-CoV-2 inhibitor as well as a randomized controlled trial to investigate the efficacy for COVID-19. These studies will shed new light on an adjuvant treatment strategy for this potentially lethal viral disease.

\section{Introduction}

The novel coronavirus (Severe Acute Respiratory Syndrome-Coronavirus-2, SARS-CoV-2) disease (COVID19) was first detected in Wuhan, China in December 2019. As the global ongoing epidemic of SARS-CoV2 , there have been more than 7,500,000 people infected and more than 40,000 patients died according to the WHO. COVID-19 is an acute infectious disease with common symptoms including fever, cough, shortness of breath, and dyspnea. In more severe cases, infection causes pneumonia, severe acute respiratory syndrome, kidney failure, or even death [1]. Although several anti-viral (mainly anti-HIV) drugs and hydroxychloroquine were investigated the efficacy for the COVID-19, the outcomes of these trials were not consistent, and no specific therapeutic drug or vaccine have been approved for COVID-19 [2].

The SARS-CoV-2 belongs to the $\beta$ genus of coronavirus which includes four genera: $a, \beta, \gamma$, and $\delta$. Coronavirus are enveloped with a positive RNA genome. Potential anti-coronavirus therapies could act on the human immune system/cells or the coronavirus. The latter therapies include blocking the specific genes of viral RNA synthesis, inhibiting critical enzymes of virus replication, inhibiting structural proteins of virus' self-assembly process, and blocking the binding between virus and human cell receptors [1]. Non-structure proteins (Nsps) involve in viral RNA transcription, RNA translation, protein synthesis, protein processing and modification, virus replication, and infection of the host [1]. In Nsps, 3-chymotrypsin-like protease (3CLpro, also named as $\mathrm{M}^{\mathrm{pro}}$ ) is automatically cleaved from polyproteins to produce mature enzymes, and further cleaves downstream Nsps at 11 sites to release Nsp4-16 [3]. The previous study on 
structure and catalytic mechanism of SARS-CoV $\mathrm{M}^{\text {pro }}$ allows it as an important target for anti-coronavirus drug development.

There are three strategies for developing an anti-SARS-CoV-2 drug [4]. The first is to test existing broadspectrum antivirals for their metabolism, used dosages, efficacy and side effects are clear. However, broad-spectrum antivirals cannot kill SARS-CoV-2 with clear targeting and the side effects should not be underestimated [1]. The second is to high-throughput-screen for SARS-CoV-2 therapeutic candidates from existing clinical drugs ("old drugs") [5, 6]. The third is to develop a new drug from scratch, however, the development and registration procedure of a new antiviral would be theoretically time-consuming [7]. For the novel virus, screening of potential "old drug" molecules for COVID-19 would be the fastest way. Since old drugs have been prepared, the medication has sufficient experience, and the safety and pharmacokinetic parameters are well known, with the in vivo efficacy in animal model, it could be approved by the Green Channel or the hospital ethics committee for clinical use [1].

In this study, we established a small-scale "old" drug database (clinical drugs being used in Ordos Central Hospital and existing antivirals) according to Linpinskis Rule of 5, and conducted in silico screening of potential $\mathrm{M}^{\text {pro }}$ inhibitors from the database by molecular docking. Binding affinity and interaction as well as structure-affinity relationship were analyzed to better understand the potentiality. Finally, literature support for the potentiality of anti-SARS-CoV-2 and the treatment of COVID-19 was reviewed and analyzed. This study will provide contributions to the transient ongoing infectious disease.

\section{Materials And Methods}

\subsection{Pharmacophore study of the co-crystal ligand N3}

The crystal structure of SARS-CoV-2 $\mathrm{M}^{\text {pro }}$ in complex with a designed small ligand N3 to 2.1- $\AA$ resolution (PDB code: 6LU7) had been determined by Professors Zihe Rao and Haitao Yang's research team from ShanghaiTech University [8]. The protein coordinates of the $\mathrm{M}^{\mathrm{pro}}$ used in this study were in-time donated by Zihe Rao et al. Based on the structure, key helixes/loops, amino acid residues, and hydrophobic interactions in binding site were investigated and the pharmacophore of N3 was summarized, which was used as a control in the following in silico study.

\subsection{Small-scale drug database}

According to Linpinskis Rule of 5 (e.g., molecular mass less than $500 \mathrm{Da}$, no more than 5 hydrogen bond donors, no more than 10 hydrogen bond acceptors, and an octanol-water partition coefficient not greater than 5) [9], we established a small-scale database including 115 common-available drugs clinically being used in Ordos Central Hospital and 20 existing antivirals. Requirement-reached drug 2D structures were drawn by ChemDraw Professional 17.0 software (CambridgeSoft Corporation, Cambridge, MA, USA). The 2D structures of candidates were converted into 3D structural data by Chem3D Ultra 17.0 software (CambridgeSoft Corporation, Cambridge, MA, USA), and all structures of the ligands were energyminimized. 


\subsection{Molecular docking by AutoDock Vina}

We applied a workflow for molecular docking which was described in our previous work [10]. The chain B (co-crystal ligand N3 in 6LU7) and chain C (water molecules) were deleted, and chain A was prepared for docking within the molecular modeling software package Chimera 1.10.2 (National Institutes of Health, Bethesda, MD, USA) [11]. Adding of polar hydrogens and kollman charges, gasteiger computing and grid box parameters defining were done using MGL tools 1.5.6 (The Scripps Research Institute, La Jolla, CA, USA) $[12,13]$.

All the ligands were set as flexible and the receptor was set as rigid. Docking calculations were performed using AutoDock Vina 1.1.2 software (The Scripps Research Institute, La Jolla, CA, USA) [14]. A search grid box was set to cover the whole surface of $\mathrm{M}^{\text {pro }}$ protein to collect all possible orientations and conformations of the ligand paired with the protein (including compounds outside the active site). For which, the center was set as: center_x $=-23.982$, center_y $=12.114$, center_z $=57.466$, and the size was set as: size_ $x=58$, size_y $=78$, size_z $=66$. Spacing angstrom was set as 1.000 , and the exhaustiveness was set as 100 . The default settings and the AutoDock Vina scoring function were applied.

Totally 9 binding modes were generated by AutoDock Vina for each compound, and the mode (even outside the active site) with highest binding affinity was selected as the most predictable. Visual investigation and analysis of ligand-protein interactions were performed using PyMOL V.1.5 (Schrodinger LLC, New York, NY, USA). Binding conformation, affinity, and receptor-ligand interaction were analyzed with $\mathrm{N} 3$ as a control.

\subsection{Re-docking study by Discovery Studio}

The accurate 3D protein structure of $\mathrm{M}^{\mathrm{pro}}$ was defined as the receptor and optimized by hydrogenation, dehydration and removing redundant residues. Location of the originally contained ligand N3 in the cocrystal was defined as the active binding site with radius as 13.890841 which could cover the best binding region. The $X, Y$, and $Z$ centers were $-10.797,12.536$, and 68.905 , respectively. Molecular structure of N3 was also prepared and converted to 3D structure and its energy was minimized. The molecular docking was performed using CDOCKER tool. -CDOCKER_ENERGY and CDOCKER_INTERACTION_ENERGY was used to score the interaction between receptor and ligand. Discovery Studio 2016 software (Biovia, San Diego, CA, USA) was used for the docking, visualization, and analysis.

\subsection{Structure-affinity relationship of promising drug}

Considering certain molecular structure of a ligand underlies certain ligand-receptor binding conformation, the generated information is relatively limited. Investigation of promising drug analogues might provide further information. After the in silico screening and the re-docking simulation of promising drug, the analogues were collected by referring to literatures from PubMed, Elsevier, Springer, and Google Scholar, and a small-scale analogue database was established. Then one-by-one docking of the 
analogues targeting on $\mathrm{M}^{\text {pro }}$ was performed. Based on the analogues' binding affinities, the structureaffinity relationship of the promising drug was summarized.

\subsection{Literature review of promising drug}

Considering anti-SARS-CoV-2 potentiality of the promising drug is simulation, we further questioned whether the promising drug have documented antiviral activities to the virus genera and whether the promising drug possess biological activities associated with the pathological changes in COVID-19 condition. We searched PubMed, Elsevier, Springer, and Google Scholar for articles describing SARS-CoV2 virus, COVID-19 condition, and the biological changes during the promising drug use. The effect of COVID-19 and the promising drug on human biological changes were summarized and analyzed.

\section{Results}

\subsection{Pharmacophore of the co-crystal ligand N3}

As illustrated in Fig. 1, M $\mathrm{M}^{\mathrm{pro}}$ monomer has three domains: domain $\otimes$ (residues 8-101, 6 antiparallel $\beta$ sheet), domain $\otimes$ (residues 102-184, 6 antiparallel $\beta$-sheet) and domain $\otimes$ (residues 201-303, 5 a-helixes,

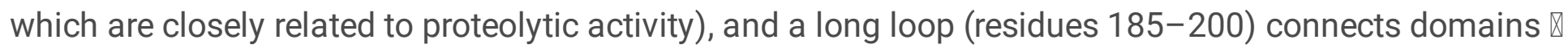
and $\otimes$. A highly conserved substrate-binding pockets (with a Cys145-His41 catalytic dyad) located in a

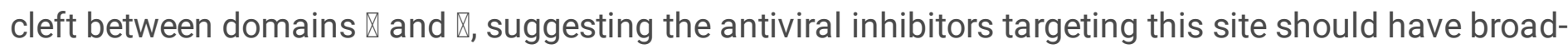
spectrum anti-coronavirus activity [8].

As shown in the diagram of Fig. 1, a covalent bond between the SY atom of Cys145 and the C $\beta$ of the vinyl group is formed, which means the Michael Addition that is critical in the catalytic mechanism has occurred [3]. The lactam functional group at P1 site inserts into the subsite S1 (consists of residues Phe140, Asn142, Glu166, His163, His172, and Leu141 as well as two waters) and forms a hydrogen bond with His163, while the functional group Leu at P2 site inserts deeply into the hydrophobic subsite S2 (consists of residues His41, Met49, Tyr54, Met165, and Asp187) [8]. The functional group Val at P3 site is solvent-exposed tolerating a variety of functional group substitutions. The functional group Ala at P4 side is in a hydrophobic pocket (consists of residues Met165, Leu167, Phe185, GIn189, and GIn192). P5 site makes van der Waals interactions with Pro168, Thr190, and Ala191, while the hydrophobic aromatic ring of N3 forms van der Waals contacts with Thr24 and Thr25. Besides, N3 forms multiple hydrogen bond interactions with the active site residues, helping to lock the inhibitor inside the binding pocket, which determines the inhibition of the enzyme as well as the coronavirus replication [8].

\subsection{In silico screening by AutoDock Vina}

For validation of docking simulation, N3 was re-docked into $\mathrm{M}^{\text {pro }}$ [10]. The docking workflow (described in 2.3) allowed top-ranked and reproduced binding conformation which was close to those of the 6LU7 cocrystal structure (checked by PyMOL, RMSD of $1.126 \AA$ ). According to AutoDock Vina, binding affinity $\leq$ $-0.0 \mathrm{kcal} / \mathrm{mol}$ means the receptor and ligand could automatically bind together. In this study, molecule 
with binding affinity $\leq-8.5 \mathrm{kcal} / \mathrm{mol}$ was treated to be potential based on recent reports on in silico screening of SARS-CoV-2 $\mathrm{M}^{\text {pro }}$ inhibitors [15].

All the 135 "old" drug structures, biological activities, targets, and top-ranked binding affinities were summarized (Supporting information Table. S1). In which, 6 molecules including anti-HIV drug (raltegravir), antibacterial drugs (cefonicid, cefoperazone, minocycline), and antidiabetic drugs (canaglifozin, glyburide) showed high affinities $(\leq-8.5 \mathrm{kcal} / \mathrm{mol}$ ) as well as interesting binding conformations (bound to the $\mathrm{M}^{\text {pro }}$ active site and formed interesting interactions with key residues). In particular, the antibiotic minocycline, an inhibitor of bacterial ribosomal rRNA, showed the highest binding affinity $(-9.6 \mathrm{kcal} / \mathrm{mol})$ compared with $\mathrm{N} 3(-7.7 \mathrm{kcal} / \mathrm{mol})$. The results indicated that these small molecular drugs might be $\mathrm{M}^{\text {pro }}$ inhibitors of SARS-CoV-2. However, the docking simulation was just potential, evaluations by in vitro cell-based models and in vivo animal models would provide further information whether these drugs could be used as SARS-CoV-2 inhibitors.

\subsection{Re-docking of N3 and minocycline by Discovery Studio}

To gain further validation of the docking simulation, re-docking of known ligand with the target and comparison of docking results with the published co-crystal as well as the comparison of docking results generated by different software are academically consensus.

From the CDOCKER results generated by Discovery Studio, N3 (Fig. 2A) formed conventional hydrogen bonds with residues Phe140, His163, His164, Glu166, Gln189, and Thr190. The isoxazole group formed Pi-Alkyl interaction with Ala191 and Pro168, and hydrophobic aromatic ring formed van der Waals' forces with residues Thr24, Thr25, Leu27 and Cys145. In addition, N3 molecule forms covalent bonds with multiple residues of $\mathrm{M}^{\text {pro }}$. The docking results were closely consistent with the co-crystal structure, indicating that the CDOCKER docking model was validated and suitable for in silico screening of $\mathrm{M}^{\text {pro }}$ inhibitors.

For minocycline (Fig. 2B), it contains multiple hydrophilic groups which formed conventional hydrogen bonding networks with key residues Phe140, Gly143, Cys145, His164, and Glu166 in the active site. The hydrophobic aromatic rings formed van der Waals' forces with multiple amino acid residues of $\mathrm{M}^{\mathrm{pro}}$. It is commonly accepted that covalent bond formed between the Cys145-His41 catalytic dyad and the designed compound would increase the $\mathrm{M}^{\text {pro }}$ inhibition potency, resembling the intermediate during substrate cleavage [3]. Beside the hydrogen bond between Cys145 and 2-carboxamide, a critical Pi-Cation formed between His41 and 4-dimethylamino group, which strongly supported minocycline as a Michael Addition acceptor binding with the exact catalytic site to inhibit $\mathrm{M}^{\mathrm{pro}}$. These results indicated that the multiple including critical interactions stabilized minocycline bound with $\mathrm{M}^{\text {pro }}$ in a low-energy state, which was required for $\mathrm{M}^{\mathrm{pro}}$ selection and antiviral activity.

\subsection{Structure-affinity relationship of minocycline}


Minocycline analogues were collected by referring to the literatures from PubMed, Elsevier, Springer, and Google Scholar. Finally a 44-compound (in which 21 compounds were clinical drugs) small-scale database was established. After molecular docking targeting on $\mathrm{M}^{\text {pro, }}$, the chemical structures and topranked binding affinities of the minocycline analogues were summarized (Supporting information Table. S2).

Indeed, minocycline showed a promising highest binding affinity among all the 44 analogues. Structures simply derivable from minocycline (containing the main octahydrotetracene-2-carboxamide skeleton) were analyzed and the structure-affinity relationship was summarized (Fig. 3). From the structure-affinity relationship, carbonyl functional groups should be kept and the middle hydroxyl group might be better if changed to be carbonyl. Furthermore, the terminal 2-carboxamide could be modified with moderate (not too long) moiety. On the 4,7-bis(dimethylamino) side, 4-dimethylamino group is critical for the high affinity, and the $S$-stereochemistry of $\mathrm{C} 4$ is better than the $R$-stereochemistry, which was also verified from the previous binding mode investigation that it could form the key covalent bond with His41.

\subsection{Literature review of minocycline}

Due to the limited resource, we could not test the antiviral activities of minocycline in SARS-CoV-2 cell and animal models. Instead, we did the literature review to find the support information. From PubMed, Elsevier, Springer, and Google Scholar databases, articles describing COVID-19 and minocycline use until 2020, Mar 6th were searched. Totally 165 papers including published and preprints were found, in which 25 papers were associated with the biochemical indexes of COVID-19 patients, and 22 papers were associated with the anti-inflammatory effect of minocycline. The effect of COVID-19 condition and minocycline on selected biomarkers including erythrocyte sedimentation rate (ESR), C-reactive protein (CRP), lactose dehydrogenase (LD), D-dimer, CD4 + T cell, CD8 + T cell, tumor necrosis factor (TNF)-a, interferon (IFN)-y, interleukin (IL)-6, and IL-10 were summarized and analyzed (Table 1).

A variety of clinical data confirmed that the inflammatory factor "storm" (IFS) existed and played an important role in severe or fast-progressive COVID-19 condition. Among the selected biomarkers, the increase of ESR, CRP, IL-6, and D-dimer helps to judge the COVID-19 progression [15]. Besides, the counts of lymphocytes, total T cells, CD $4+T$ cells, and CD8 $+T$ cells were dramatically reduced, while patients in decline period showing decreased levels of TNF-a, IFN- $\gamma$, IL-6, IL-10 and restored counts of T cell $[16,17]$. IL- 6 and IL-10 are found to be the core cytokines that are consistently found to be elevated in patients with CRS $[18,19]$. The increases of IL- 6 and IL-10 levels in COVID-19 patients were found to run parallel with the severity of the disease [20]. Consistent decrease of CD4 + T and CD8 + T cells, spleen damage, and lymphocyte depletion exist in COVID-19 patients [21]. In severe COVID-19 patients, the elevation of CRP level and white blood cell count might be accompanying with bacterial infection, and antibiotics were sometimes prescribed [22]. 
Table 1

Comparison of the effect of COVID-19 and minocycline on selected biomarkers.

\begin{tabular}{|c|c|c|c|c|}
\hline \multirow[t]{2}{*}{$\begin{array}{l}\text { Biological } \\
\text { Markers }\end{array}$} & \multicolumn{2}{|l|}{ COVID-19 } & \multicolumn{2}{|c|}{$\begin{array}{l}\text { Minocycline Effect on Physical or } \\
\text { Infectious Challenge }\end{array}$} \\
\hline & Severe (References) & $\begin{array}{l}\text { Non-severe } \\
\text { (References) }\end{array}$ & $\begin{array}{l}\text { In Vivo } \\
\text { (References) }\end{array}$ & $\begin{array}{l}\text { In Vitro } \\
\text { (References) }\end{array}$ \\
\hline \multirow[t]{2}{*}{ ESR } & $\uparrow([23] ;[21] ;[24])$ & $\uparrow([21] ;$ & $\downarrow([32] ;[33] ;[34])$ & \\
\hline & & [24]) & & \\
\hline CRP & $\begin{array}{l}\uparrow([21] ;[25] ;[[26] ;[22] ; \\
\text { [27]; [28]; [29]) }\end{array}$ & $\begin{array}{l}\uparrow([21] ;[25] ;[26] ; \\
\text { [22]; [27]) }\end{array}$ & $\begin{array}{l}\downarrow l([35] ; ;[34] ;[36] ; \\
\text { [37]; [38]) }\end{array}$ & \\
\hline LD & $\uparrow([21] ;[26] ;[30] ;[31])$ & $\uparrow([21])$ & & $\downarrow([26])$ \\
\hline D-dimer & 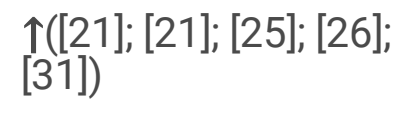 & $\uparrow([21] ;[26])$ & $\downarrow([39])$ & \\
\hline $\mathrm{CD} 4+\mathrm{T}$ cell & $\downarrow([16] ;[17])$ & $\downarrow([17] ;[24])$ & $\uparrow([40])$ & \\
\hline $\mathrm{CD} 8+\mathrm{T}$ cell & $\downarrow([16] ;[17])$ & $\downarrow([17] ;[24])$ & $\uparrow([40])$ & \\
\hline TNF-a & $\uparrow([16])$ & & $\begin{array}{l}\downarrow([41] ;[42] ;[43] ; \\
[44])\end{array}$ & $\downarrow([45] ;[46] ;[47])$ \\
\hline IFN- $\gamma$ & $\uparrow([16] ;[17])$ & $\uparrow([17])$ & & $\downarrow([47] ;[48])$ \\
\hline IL-6 & $\uparrow([21] ;[25] ;[16] ;[17])$ & $\uparrow([17] ;[24] ;[21])$ & $\downarrow([41] ;[42] ;[43])$ & $\downarrow([49] ;[47])$ \\
\hline IL-10 & $\uparrow([16] ;[17])$ & $\uparrow([17] ;[24])$ & & $\begin{array}{l}\perp([41] ;[45] ;[50] ; \\
[47])\end{array}$ \\
\hline $\begin{array}{l}\text { ESR, erythroc } \\
\text { necrosis fact } \\
\text { decreased. }\end{array}$ & $\begin{array}{l}\text { e sedimentation rate; } \\
\mathrm{a} ; \text { IFN-y, Interferon } \gamma ; \text {; I }\end{array}$ & $\begin{array}{l}\text {-reactive protein; } \\
\text { erleukin; } \uparrow \text {, signific }\end{array}$ & $\begin{array}{l}\text { lactose dehydrc } \\
\text { tly increased; } \downarrow \text {, }\end{array}$ & $\begin{array}{l}\text { se; TNF-a, tumor } \\
\text { cantly }\end{array}$ \\
\hline
\end{tabular}

It is critical to control the IFS in COVID-19, as shown in Table 1, the levels of broad-spectrum of selected biological markers associated with minocycline administration were opposed to those of COVID-19 condition (both severe and non-severe). In terms of TNF-a, IFN- $y$, IL-6 and IL-10, the affected levels by minocycline on physical or infectious challenge obviously opposite to those in COVID-19 condition, which was strongly supported by in vivo and in vitro data. These findings suggested that minocycline, a safe, inexpensive, and readily available antibiotic, could be considered as an adjunctive therapeutic option for severe and fast-progressive COVID-19 patients.

\section{Discussion}

\subsection{In silico screening of SARS-CoV-2 $M^{\text {pro }}$ inhibitors}

There is still no approved specific therapy for the ongoing COVID-19. For the treatment, screening of potential "old drug" molecules for COVID-19 would be the fastest way. Since old drugs have been 
prepared, the medication has sufficient experience, and the safety and pharmacokinetic parameters are well known, with the in vivo efficacy in animal model, it could be approved by the Green Channel or the hospital ethics committee for clinical use [1].

$\mathrm{M}^{\text {pro, }}$, which is highly conserved among all coronavirus, is a good target for the development of a single antiviral agent or in combination with other potential therapies to provide an effective first line of defense against all coronavirus-associated diseases [8]. The co-crystal structure of SARS-CoV-2 $\mathrm{M}^{\text {pro }}$ complexed with N3 is a good model for identifying inhibitor lead through in silico screening. Based on the "New uses of old drugs" research concept, 135 old drugs were screened targeting on $\mathrm{M}^{\text {pro }}$ in this study. A group of "old" drugs were screened out, in which the antibiotic drug minocycline showed highest affinity. Docking simulation and the structure-affinity relationship study found critical covalent bond formed between the active Cys145-His41 catalytic dyad and minocycline, which helped us to better understand why the functional groups as well as the tetracycline skeleton could be suitable for the $\mathrm{M}^{\mathrm{pro}}$ active-site binding and interaction. The in silico screening is just simulation, in vitro and in vivo anti-SARS-CoV-2 evaluations of minocycline will provide useful information.

\subsection{Biological activities of minocycline}

Minocycline is an FDA-approved, second-generation tetracycline class antibiotic with an established safety profile that has been used in clinic for more than 30 years. Minocycline binds to the bacterial $30 \mathrm{~S}$ ribosomal subunit, inhibiting the binding of RNA to ribosomes, and interferes with protein synthesis [51]. The main associated pharmacological conditions of minocycline were both gram-positive/negative bacterial infections and the more recent multidrug resistant Acinetobacter baumannii [52]. In spite of this, minocycline appears to have broad-spectrum antiviral activities: reducing West Nile Virus titers in brainderived cell types, reducing Japanese encephalitis-induced damage in neuronal cells inhibiting H7N9 replication in human lung epithelial cells, and attenuating pathogenic immune responses during infection with human and simian immunodeficiency virus (HIV/SIV) [48, 53-56]. Moreover, based on molecular docking and dynamic studies, minocycline was proposed as potential antiviral therapy against Congo Crimean hemorrhagic fever virus to inhibit the binding of virus to host nucleoprotein [57]. In a randomized controlled trial of dengue hemorrhagic fever patients, compared with standard-of-care, combination therapy with doxycycline (analogue of minocycline) significantly decreased the TNF and IL-6 levels, and mortality [58].

Literatures showed that the broad anti-IFS activity of minocycline opposed those of many biological markers of COVID-19 condition. In addition, ACE2 (the functional receptor for SARS-CoV-2) is present in multiple human organs including nervous system and skeletal muscle [59]. Severe COVID-19 patients were more likely to develop neurological symptoms [28]. Minocycline attenuates T cell and microglia activity to impair cytokine production in T cell-microglia interaction [45]. Due to the small size and lipophilic nature, minocycline might cross into tissue compartments with potentially therapeutic concentrations.

\subsection{IFS-suppressing treatment of COVID-19}


IFS is an inappropriate immune response that is caused by rapidly proliferating and highly activated $T$ cells, more than 100 inflammatory factors are released, and subsequently lead to tissue damage and organ failure [60]. Inflammatory responses triggered by viral infection play a crucial role in pulmonary pathology severity [61]. Suppressing the IFS to reduce lung inflammation might be a valuable treatment method. High doses of glucocorticoid were widely applied during the outbreaks of SARS and Middle East Respiratory Syndrome coronavirus infections to suppress lung inflammation and immune responses [62-64]. However, it appeared to be associated with side effects, such as secondary bacterial infection, osteoporosis, etc. Therefore, glucocorticoid was not generally recommended for severe COVID-19 due to its inhibition of immune responses and pathogen clearance [17]. However, the immune imbalance and bacterial infection often appear in the later stages of COVID-19 progression, the efficacy of antiviral drugs might remain unsatisfactory or insufficient [2]. The antibiotics and glucocorticoid were sometimes administered according to the clinical characteristics and physicians' discretion [31].

Adjunctive therapy for anti-IFS but not globally downregulate the host immune response may hold promise for better outcomes of COVID-19. Thalidomide, an immunomodulatory and anti-inflammatory agent, was case-reported the protective effect on lung injury and immunological stress caused by COVID19 pneumonia in combination with antiviral drugs and low-dose glucocorticoid [17]. The acute pulmonary effusion symptoms and the elevated inflammatory cytokine profiles including IFN-y, IL-6 and IL-10 were reduced, and the number of lymphocytes recovered [65]. In a two-year randomized controlled trial on early seropositive rheumatoid arthritis patients, minocycline achieved better anti-inflammatory outcomes than hydroxychloroquine [66]. Chloroquine was included in the 6th version of Diagnostic and Treatment Protocol for COVID-19 in China due to the in vitro anti-SARS-CoV-2 results and in vivo anti-inflammatory activity. In case of hydroxychloroquine, it is chemically and biologically similar but safer than chloroquine, and has been suggested by scholars and included in the Shanghai local diagnostic and treatment guidelines for COVID-19 [66,67]. Whether minocycline is better than chloroquine, hydroxychloroquine and thalidomide in COVID-19 deserves a randomized controlled trial.

\subsection{Minocycline as an adjunctive COVID-19 therapy option}

Based on the above findings, we concluded that minocycline could be considered as an adjunctive therapy option for the treatment as well as the chronic sequela of COVID-19. For one thing, it has anti-IFS activity against biomarkers that appear to be pathologically changed in COVID-19 condition. For another, it could prevent or treat the bacterial/mycoplasma infection along with COVID-19 progression. The most important point is that it has broad-spectrum antiviral activities and potential anti-SARS-CoV-2 activity. Chloroquine (hydroxychloroquine), steroids, and high-dose of urinastatin have been proposed in several COVID-19 treatment guidelines in China for the anti-inflammatory consideration. Minocycline might be a good alternative/adjunctive option of the proposed drugs, and it is likely that the anti-IFS benefit would be more significant in severe and fast-progressive infected populations. Anyway, in vitro and in vivo evaluations of minocycline as SARS-CoV-2 inhibitor as well as a randomized controlled trial to investigate the efficacy for COVID-19 treatment will shed new light on an adjuvant treatment strategy for this potentially lethal viral disease. 


\section{Conclusion}

In conclusion, we did an in silico screening of 135 clinical drugs targeting on $\mathrm{M}^{\text {pro }}$ of the novel SARS-CoV2 , and the antibiotic minocycline, an inhibitor of bacterial ribosomal rRNA, showed the highest binding affinity $(-9.6 \mathrm{kcal} / \mathrm{mol})$. Valuable hydrogen bonding including critical bonds with the Cys $145-H i s 41$ catalytic dyad and hydrophobic interactions were found between minocycline and $\mathrm{M}^{\text {pro }}$ active site. The structure-affinity relationship study helped to explain the conformation suitability of minocycline. Literature review found that minocycline had both in vitro and in vivo broad-spectrum antiviral as well as anti-inflammatory activities, and the levels of a broad-spectrum of biological markers during minocycline administration were opposed to those of COVID-19 condition. Minocycline deserves in vitro and in vivo evaluations as SARS-CoV-2 inhibitor as well as a randomized controlled trial to investigate the efficacy for COVID-19. These studies will shed new light on an adjuvant treatment strategy for this potentially lethal viral disease.

\section{Declarations}

\section{Acknowledgements}

We thank Professors Zihe Rao, Haitao Yang, and Xiuna YangfromShanghaiTech University for the in time donation of $\mathrm{M}^{\text {proinformation. }}$

\section{Author contributions}

Guanhua Du, Fengxiang Zhang, and Zhanfei She conceptualized and supervised this study. Bin Xiao, Haining Ning, Jinhua Wang, andLiwen Ren designed the research, performed virtual screening. Bin Xiao, Haining Ning, Yiqing Wang, Liwen Ren, Jinhua Wang,Zhong Wang, and Xiangjin Zheng analyzed the docking results analysis. Bin Xiao and YixuanNiu designed and carried out theliterature review. All authors revised the manuscript, and have read and approved the final manuscript.

\section{Funding}

This work was supported by the National Science Foundation for Post-doctoral Scientists of China (2019M650537), the Natural Science Foundation of Inner Mongolia (2017BS0808), and the Ordos Innovation Fund for Talent Team (Ordos Talent Work Leading Group2018-No.6, China).

\section{Declarations}

The authors declare that they have no conflict of interest.

\section{References}

[1]Wu,C.R., Liu, Y., Yang, Y. Y.,Zhang, P., Zhong, W., Wang, Y., et al. (2020). Analysis of therapeutic targets for SARS-CoV-2 and discovery of potential drugs by computational methods, Acta Pharm Sin B., 
27;10(5):766-788. doi: 10.1016/j.apsb.2020.02.008.

[2]Huang,C.L., Wang, Y. M., Li, X. W., Ren, L. L. , Zhao, J. P., Hu, Y.,et al. (2020). Clinical features of patients infected with 2019 novel coronavirus in Wuhan, China. Lancet, 395, 15-21.doi: 10.1016/S01406736(20)30183-5.

[3]Yang,H.T., Xie, W. Q., Xue, X. Y., Yang, K. L.,Ma, J. , Liang, W. X., et al. (2005).Design of wide-spectrum inhibitors targeting coronavirus main proteases.PLoS Biol.,3,e324.doi: 10.1371/journal.pbio.0030324.

[4]Zumla,A., Chan, J., Azhar, E.,Hui, D., and Yuen, K. (2016).Coronaviruses-drug discovery and therapeutic options.Nat. Rev. Drug Discov.,15, 327-47.doi:10.1038/nrd.2015.37.

[5]Wilde,A.H., Jochmans, D., Posthuma, C. C., Zevenhoven-Dobbe, J. C., Nieuwkoop, S., Bestebroer, T. M.,et al. (2014) .Screening of an FDA-approved compound library identifies four small-molecule inhibitors of Middle East Respiratory syndrome coronavirus replication in cell culture. Antimicrob. Agents Chemother.,14, 4875-84.doi: 10.1128/AAC.03011-14.

[6]Dyall,J., Coleman, C. M., Hart, B. J., Venkataraman, T., Holbrook, M. R., Kindrachuk, J., et al. (2014).Repurposing of clinically developed drugs for treatment of Middle East respiratory syndrome coronavirus infection.Antimicrob. Agents Chemother., 58, 4885-93. doi: 10.1128/AAC.03036-14.

[7]Omrani,A.S.Saad, M. M., Baig, K., Bahloul, A., Abdul-Matin, M.,Alaidaroos, A.Y., et al. (2014). Ribavirin and interferon alfa-2a for severe Middle East respiratory syndrome coronavirus infection: a retrospective cohort study. Lancet Infect. Dis., 14, 1090-1095.doi: 10.1016/S1473-3099(14)70920-X.

[8]Jin,Z.M., Du, X. Y., Xu, Y. C., Deng, Y. Q., Liu, M. Q., Zhao, Y., et al. (2020).Structure of Mpro from COVID19 virus and discovery of its inhibitors. Nature $5582(7811)$ :289-293.doi: 10.1038/s41586-020-2223-y.

[9]Lipinski,C.A., Lombardo, F., Dominy, B. W., and Feeney, P. J. (1997).Experimental and computational approaches to estimate solubility and permeability in drug discovery and development settings.Adv. Drug Deliv. Rev., 23, 3-25.doi: 10.1016/s0169-409x(00)00129-0.

[10]Xiao, B., Wang, S.M., She, Z.F., Cao, Q.F., Zhao, N., Tian, X.R., et al. (2017) Structure-based design, synthesis, PPAR-y activation, andmolecular docking of $N$-substituted phthalimides. Med. Chem. Res., 26, 1628-1634. doi:10.1007/s00044-017-1867-0.

[11]Pettersen,E.F., Goddard, T. D., Huang, C. C., Couch, G. S., Greenblatt, D. M., Meng, E. C., et al. (2004) UCSF Chimera-a visualization system for exploratory research and analysis. J.Comput. Chem., 25, 16051612.doi: $10.1002 /$ jcc.20084.

[12]Sanner,M.F. (1999) Python: a programming language for software integration and development.J. Mol. Graphics Mod., 17, 57-61. PMID: 10660911 
[13]Morris,G.M., Huey, R., Lindstrom, W., Sanner, M. F.,Belew, R. K.,Goodsell, D. S., et al.(2009).AutoDock4 and AutoDockTools4: Automated docking with selective receptor flexibility. J Comput Chem., 30, 27852791.doi: 10.1002/jcc.21256.

[14]Trott,O. and Olson,A.J. (2010) AutoDockVina: improving the speed and accuracy of docking with a new scoring function, efficient optimization, and multithreading. J.Comput.Chem., 31, 455-461.doi: $10.1002 /$ jcc. 21334 .

[15]Zong, Y., Ding, M. L., Jia, K. K., Ma, S.T., Ju, W. Z.(2020).Exploring active compounds of Da-Yuan-Yin in treatment of COVID-19 based on network pharmacology and molecular docking method.

Chin. Tradit.Herbal. Drugs, 51, 836-844. doi:10.7501/j.issn.0253-2670.2020.04.002.

[16]Diao,B.,Wang, C. H., Tan, Y. J., Chen, X. W., Liu, Y., Ning, L. F., et al. (2020). Reduction and functional exhaustion of T cells in patients with coronavirus disease 2019 (COVID-19). Front Immunol.ख11: 827.doi: 10.3389/fimmu.2020.00827.

[17]Cheng,Y.C., Luo, R., Wang, K., Zhang, M., Wang, Z. X., Dong, L.,et al. (2020). Kidney impairment is associated with in-hospital death of COVID-19 patients. Kidney Int., 97(5):829-838. doi:

10.1016/j.kint.2020.03.005.

[18]Tanaka,T., Narazaki, M.,and Kishimoto, T. (2014) IL-6 in inflammation, immunity, and disease.Cold SpringHarb.Perspect.Biol. 6, a016295.doi: 10.1101/cshperspect.a016295.

[19]Shimabukuro-Vornhagen,A., Gödel, P., Subklewe, M.,Stemmler, H. J., Schlößer, H. A., Schlaak, M., et al. (2018). Cytokine release syndrome, J.Immuno. Ther.Cancer,6, 56.doi: 10.1186/s40425-018-0343-9.

[20]Wan,S., Yi, Q., Fan, S., Lv, J., Zhang, X., Guo, L., et al. (2020). Characteristics of lymphocyte subsets and cytokines in peripheral blood of 123 hospitalized patients with 2019-nCoV pneumonia (NCP).medRxiv, 2020.02.10.20021832; doi: https://doi.org/10.1101/2020.02.10.20021832.

[21]Cai,Q.X., Huang, D. L., Ou, P. C., Yu, H., Zhu, Z. B., Xia, Z., et al. (2020).COVID-19 in a Designated infectious diseases hospital outside Hubei Province, China. Allergy[2020;00:1-11.doi: 10.1111/all.14309.

[22]Cao,W.L., Shi, L., Chen, L.,Xu, X. M., and Wu, Z. R.(2020).Clinical features and laboratory inspection of novel coronavirus pneumonia (COVID-19) in Xiangyang, Hubei.medRxiv. 2020.02.23.20026963; doi: https://doi.org/10.1101/2020.02.23.20026963.

[23]Zheng, Y.S., Huang, Z., Ying, G. P., Zhang, X., Ye, W., Hu, Z. L., et al. (2020). Comparative study of the lymphocyte change between COVID-19 and non-COVID-19 pneumonia cases suggesting uncontrolled inflammation might not be the main reason of tissue injury.medRxiv 2020.02. 19. 20024885; doi: https://doi.org/10.1101/2020.02.19.20024885.

[24]Cai,X.L. (2020). An insight of comparison between COVID-19 (2019-nCoV disease) and SARS-CoV in pathology and pathogenesis. 2020. OSF Preprints. February 20. doi:10.31219/osf.io/hw34x. 
[25]Liu,L. and Gao,J.Y. (2020) Clinical characteristics of 51 patients discharged from hospital with COVID19 in Chongqing, China. medRxiv 2020.02.20.20025536; doi:

https://doi.org/10.1101/2020.02.20.20025536.

[26]Qin,X.W., Qiu, S. H., Yuan, Y. M., Zong, Y.,Tuo, Z., Li, J., et al. (2020). Clinical characteristics and treatment of patients infected with COVID-19 in Shishou, China (February 18, 2020). Available at SSRN: https://ssrn.com/abstract=3541147.

[27]Qian,G.Q., Yang, N.,Ding, F., Ma, A. H. Y., Wang, Z., Shen, Y., et al. (2020). Epidemiologic and clinical characteristics of 91 hospitalized patients with COVID-19 in Zhejiang, China: A retrospective, multi-centre case series. QJM: An International Journal of Medicine $\square$ Mar 17 : hcaa089. doi: 10.1093/qjmed/hcaa089.

[28]Mao,L.,Jin, H. J., Wang, M. D., Hu, Y., Chen, S.C., He, Q. W., et al. (2020). Neurological Manifestations of Hospitalized Patients with COVID-19 in Wuhan, China: a retrospective case series study. JAMA Neurol., 77, 1-9.doi: 10.1001/jamaneurol.2020.1127.

[29]Li,X.,Wang, L. W., Yan, S. N., Yang, F., Xiang, L. K., Zhu, J. L., et al. (2020). Clinical characteristics of 25 death cases infected with COVID-19 pneumonia: a retrospective review of medical records in a single medical center, Wuhan, China. Int. J. Infect. Dis. ,May;94:128-132.doi: 10.1016/j.ijid.2020.03.053.

[30]Wang,D.W., Hu, B., Hu, C., Zhu, F. F., Liu, X., Zhang, J., et al. (2020). Clinical characteristics of 138 hospitalized patients with 2019 novel coronavirus-infected pneumonia in Wuhan, China. JAMA, 323, 1061-1069. doi: 10.1001/jama.2020.1585.

[31]Ji,D., Zhang, D. W., Chen, Z.,Xu, Z., Zhao, P.,Zhang, M. J.,et al. (2020).Clinical characteristics predicting progression of COVID-19 (2/17/2020). Available at SSRN: https://ssrn.com/abstract=3539674.

[32]Tilley,B.C., Alarcón,G. S., Heyse, S.P., Trentham, D. E.,Neuner, R. , Kaplan, D. A.,et al. (1995).for the MIRA Trial Group. Minocycline in rheumatoid arthritis: A 48-week, double-blind, placebo-controlled trial. Ann. Intern. Med., 122, 81-89.doi: 10.7326/0003-4819-122-2-199501150-00001.

[33]O'Dell,J.R., Blakely, K. W., Mallek, J. A., Eckhoff, P. J., Leff, R. D., Wees, S. J.,et al. (2001). Treatment of early seropositive rheumatoid arthritis: A two-Year, double-blind comparison of minocycline and hydroxychloroquine. Arthritis Rheum.,44, 2235-2241.doi: 10.1002/1529-0131(200110)44:10<2235::aidart385>3.0.co;2-a.

[34]Matsuyama, A., Sakai, N., Ishigami, M., Hiraoka, H., and Yamashita, S. (2005).Minocycline for the treatment of Takayasu Arteritis.Ann. Intern. Med., 143, 394-395.doi: 10.7326/0003-4819-143-5200509060-00021

[35]Kloppenburg,M., Dijkmans, B. A., Verweij, C. L., and Breedveld, F. C. (1996). Inflammatory and immunological parameters of disease activity in rheumatoid arthritis patients treated with minocycline. Immunopharmacology, 31, 163-169.doi: 10.1016/0162-3109(95)00041-0. 
[36]Kanai,M. andShimizu,H. (2009).A case of hypertrophic pachymeningitis treated successfully with antibiotics: the remarkable effect of minocycline hydrochloride in reducing the serum C-reactive protein value. Neurol Surg., 37,673-679.

[37]Soliman,M.G., Hanaamansour, A., Hasan, W. A., and Hassaan, N. A. (2015).Protective effect of minocycline against bacterial infection-induced sickness behavior in rats. Global J.Pharmacol., 9, 0112.doi: 10.5829/idosi.gjp.2015.9.1.9232.

[38]Li,J.,Wang, X. Y., Wang, M. J., Wang, C., and Song, G. C., (2017).Analyze on the influence of minocycline combined with azithromycin on serum CRP, D-Dimer and lung function in the children with refractory mycoplasma pneumonia. Chin J Biochem Pharm., 37,102-105.doi 1 10.3969/j.issn.10051678.2017.08.042.

[39]Hou,Y., Ryu, C. H., Park, K. Y., Kim, S. M.,Jeong, C. H., and Jeun, S.(2013).Effective combination of human bone marrow mesenchymal stem cells and minocycline in experimental autoimmune encephalomyelitis mice. Stem Cell Res.Ther., 4, 77.doi: 10.1186/scrt228.

[40]Shalini,M., Pecaut, M. J., and Gridley, D. S. (2012).Analysis of minocycline as a countermeasure against acute radiation syndrome.In Vivo, 26, 743-758. PMID: 22949587.

[41]Ledeboer,A., Sloane, E. M., Milligan, E. D., Frank, M. G., Mahony, J. H., Maier, S. F., et al. (2005). Minocycline attenuates mechanical allodynia and proinflammatory cytokine expression in rat models of pain facilitation. Pain, 115, 71-83.doi: 10.1016/j.pain.2005.02.009.

[42]Masocha,W., Rottenberg, M. E., and Kristensson, K. (2006). Minocycline impedes African trypanosome invasion of the brain in a murine model. Antimicrob. Agents Ch., 50, 1798-1804.doi: 10.1128/AAC.50.5.1798-1804.2006.

[43]Suzuki,H., Sugimura, Y., Iwama, S., Suzuki, H., Nobuaki, O., Nagasaki, H.,et al. (2010). Minocycline prevents osmotic demyelination syndrome by inhibiting the activation of microglia. J. Am. Soc. Nephrol, 21, 2090-2098.doi: 10.1681/ASN.2010040438.

[44]Wu,K.L., Chan, S. H. H., and Chan, J. Y. H. (2012).Neuroinflammation and oxidative stress in rostral ventrolateral medulla contribute to neurogenic hypertension induced by systemic inflammation. J.Neuroinflamm., 9, 212. doi: 10.1186/1742-2094-9-212.doi: 10.1186/1742-2094-9-212.

[45]Giuliani,F., Hader, W., and Yong, V. W. (2005). Minocycline attenuates T cell and microglia activity to impair cytokine production in T cell-microglia interaction. $J$ Leukocyte Biol., 78,135-143.doi: 10.1189/jlb.0804477.

[46]Szeto,G.L., Brice, A. K.,Yang, H.,Barber, S. A., Siliciano,R. F., and Clements, J. E., (2010). Minocycline attenuates HIV infection and reactivation by suppressing cellular activation in human CD4+ T cells. $J$. Infect. Dis., 201, 1132-1140. doi: 10.1086/651277. 
[47]Tai,K.,Iwasaki, H.,Ikegaya, S., and Ueda, T. (2013). Minocycline modulates cytokine and chemokine production in lipopolysaccharide-stimulated THP-1 monocytic cells by inhibiting IKB kinase $\alpha / \beta$ phosphorylation. Transl. Res., 161, 99-109.doi: 10.1016/j.trsl.2012.10.001.

[48]Drewes,J.L., Szeto, G. L., Engle, E. L., Liao, Z. H., Shearer, G. M., Zink, M. C., et al. (2014).Attenuation of pathogenic immune responses during infection with human and simian immunodeficiency virus (HIV/SIV) by the tetracycline derivative minocycline.PloSOne, 9, e94375.doi:

10.1371/journal.pone.0094375.

[49]Campbell,J.H., Burdo, T. H., Autissier, P., Bombardier, J. P., Westmoreland, S. V., Soulas, C., et al. (2011). Minocycline inhibition of monocyte activation correlates with neuronal protection in SIV neuroAIDS. PloS One, 6, e18688.doi: 10.1371/journal.pone.0018688.

[50]Yang,L. P.,Zhu, X. A., and Mark, O.M. (2007). Minocycline and sulforaphane inhibited lipopolysaccharide-mediated retinal microglial activation. Mol. Vis., 13, 1083. doi:10.1016/j.visres.2007.06.007.

[51]Hong,W.L., Zeng, J., and Xie, J. P. (2014).Antibiotic drugs targeting bacterial RNAs.Acta Pharm. Sin. B, 4, 258-265.doi: 10.1016/j.apsb.2014.06.012.

[52]Lashinsky,J.N., Henig, O., Pogue, J. M., and Kaye, K. S.(2017). Minocycline for the treatment of multidrug and extensively drug-resistant a. baumannii: A review. Infect. Dis. Ther., 6, 199-211. doi: 10.1007/s40121-017-0153-2.

[53]Michaelis,M., Kleinschmidt, M. C., Doerr, H. W., and Jr, J. C. (2007). Minocycline inhibits West Nile virus replication and apoptosis in human neuronal cells. J.Antimicrob.Chemother.,60, 981-986. doi:

10.1093/jac/dkm307.

[54]Mishra,M.K.,Ghosh, D., Duseja, R.and Basu, A. (2009). Antioxidant potential of minocycline in japanese encephalitis virus infection in murine neuroblastoma cells: correlation with membrane fluidity and cell death.Neurochem.Int., 54, 464-470. doi: 10.1016/j.neuint.2009.01.022.

[55]Josset,L., Zeng, H., Kelly, S. M., Tumpey, T. M., and Katze, M. G.(2014). Transcriptomic characterization of the novel avian-origin influenza A (H7N9) virus: specific host response and responses intermediate between avian (H5N1 and H7N7) and human (H3N2) viruses and implications for treatment options. mBio.,5, e01102-e01113.doi: 10.1128/mBio.01102-13.

[56]Hendricks, K., Parrado, M. G., and Bradley, J. (2020). Opinion: An existing drug to assess in vivo for potential adjunctive therapy of Ebola Virus disease and post-Ebola syndrome. Front. Pharmacol. 10:1691. doi: 10.3389/fphar.2019.01691

[57]Sharifi,A., Amanlou, A., Moosavi-Movahedi, F., Golestanian, S., and Amanlou, M. (2017).Tetracyclines as a potential antiviral therapy against Crimean Congo hemorrhagic fever virus: Docking and molecular 
dynamic studies. Comput. Biol. Chem., 70, 1-6.doi: 10.1016/j.compbiolchem.2017.06.003.

[58]Fredeking,T.M., Zavala-Castro, J. E., González-Martínez, P., Moguel-Rodríguez, W., Sanchez, E. C., Foster, M. J., et al. (2015). Dengue patients treated with doxycycline showed lower mortality associated to a reduction in IL-6 and TNF levels. Recent patents on anti-infective drug discovery. Recent PatAntiinfect Drug Discov.,10, 51-58. doi: 10.2174/1574891x10666150410153839.

[59]Zhao,Y., Zhao, Z. X., Wang, Y. J., Zhou, Y. Q.,Ma, Y., and Zuo, W. (2020).Single-cell RNA expression profiling of ACE2, the putative receptor of Wuhan 2019-nCov.bioRxiv 2020.01.26.919985; doi: https://doi.org/10.1101/2020.01.26.919985.

[60]Teijaro,J.R., Walsh, K. B., Cahalan, S. M., Fremgen, D. M., Roberts, E., Scott, F., et al. (2011). Endothelial cells are central orchestrators of cytokine amplification during influenza virus infection. Cell, 146, 980991.doi: 10.1016/j.cell.2011.08.015.

[61]Zheng,J., and Perlman,S. (2018). Immune responses in influenza a virus and human coronavirus infections: An ongoing battle between the virus and host. Curr.Opin. Virol.,28, 4352.doi:10.1016/j.coviro.2017.11.002.

[62]Stockman,L.J., Bellamy, R.and Garner, P. (2006). SARS: systematic review of treatment effects. PLoS Med., 3, e343.doi: 10.1371/journal.pmed.0030343.

[63]Arabi,Y.M.,Mandourah, Y., Al-Hameed, F., Sindi, A. A., Almekhlafi, G. A., Hussein, M. A., et al. (2018).Corticosteroid Therapy for Critically III Patients with Middle East Respiratory Syndrome. Am. J. Resp. Crit. Care, 197, 757-67.doi: 10.1164/rccm.201706-11720C.

[64]Venkatesh,B., Finfer, S., Cohen, J., Rajbhandari, D., Arabi, Y., Bellomo, R., et al. (2018).Adjunctive Glucocorticoid Therapy in Patients with Septic Shock.N. Engl.J. Med., 378, 797-808.doi:

10.1056/NEJMoa1705835.

[65]Chen,C.S., Qi, F., Shi, K. Q., Li, Y. P., Li, J., Chen, Y. P., et al. (2020). Thalidomide combined with low-dose glucocorticoid in the treatment of COVID-19 pneumonia. Preprints, 2020020395.

[66]Wang,M.L., Cao, R. Y., Zhang, L. K., Yang, X. L., Liu, J., Xu, M. Y., et al. (2020).Remdesivir and chloroquine effectively inhibit the recently emerged novel conronavirus (2019-nCoV) in virto. Cell Res., 30, 269-271.doi: 10.1038/s41422-020-0282-0.

[67]Stokkermans,T.J. et al. (2019) In: StatPearls. StatPearls Publishing, Treasure Island (FL); 2019.

\section{Figures}




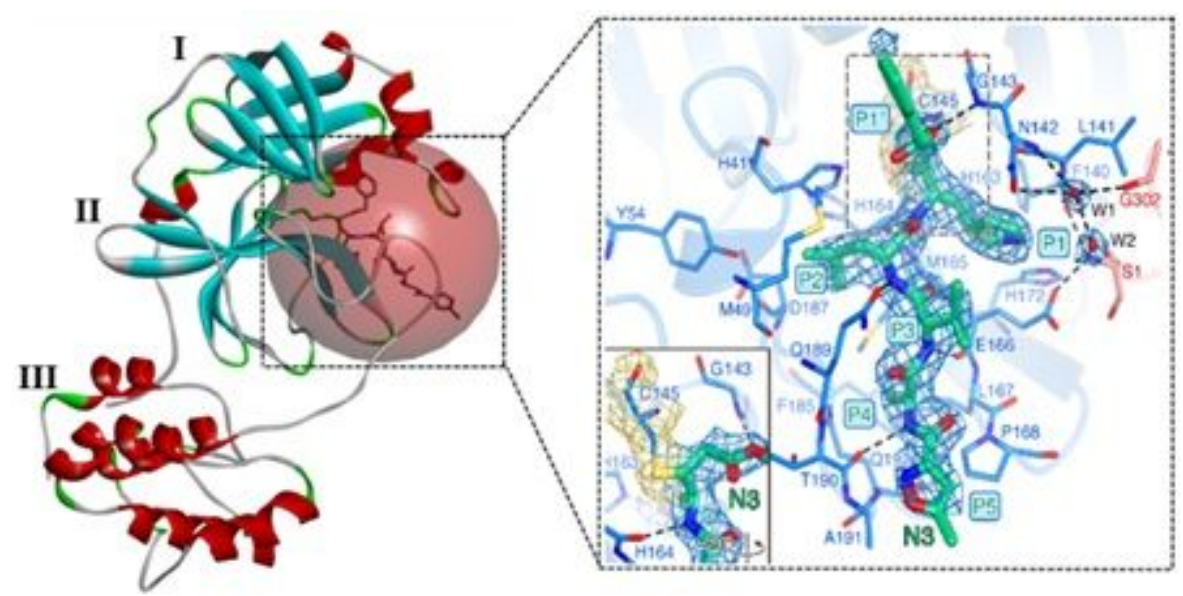

Figure 1

N3 bound in Mpro active site and the diagram of the interactions in the co-crystal.P1, P1', P2, P3, P4 and P5 sites of N3 are indicated, 2Fo-Fc density map is shown aroundN3 molecule in blue mesh, C145-A in yellow mesh, and water in blue mesh[8]. The key residue is shown in stick, hydrogen bond is shown in black dashed line, and water is shown as red sphere. 


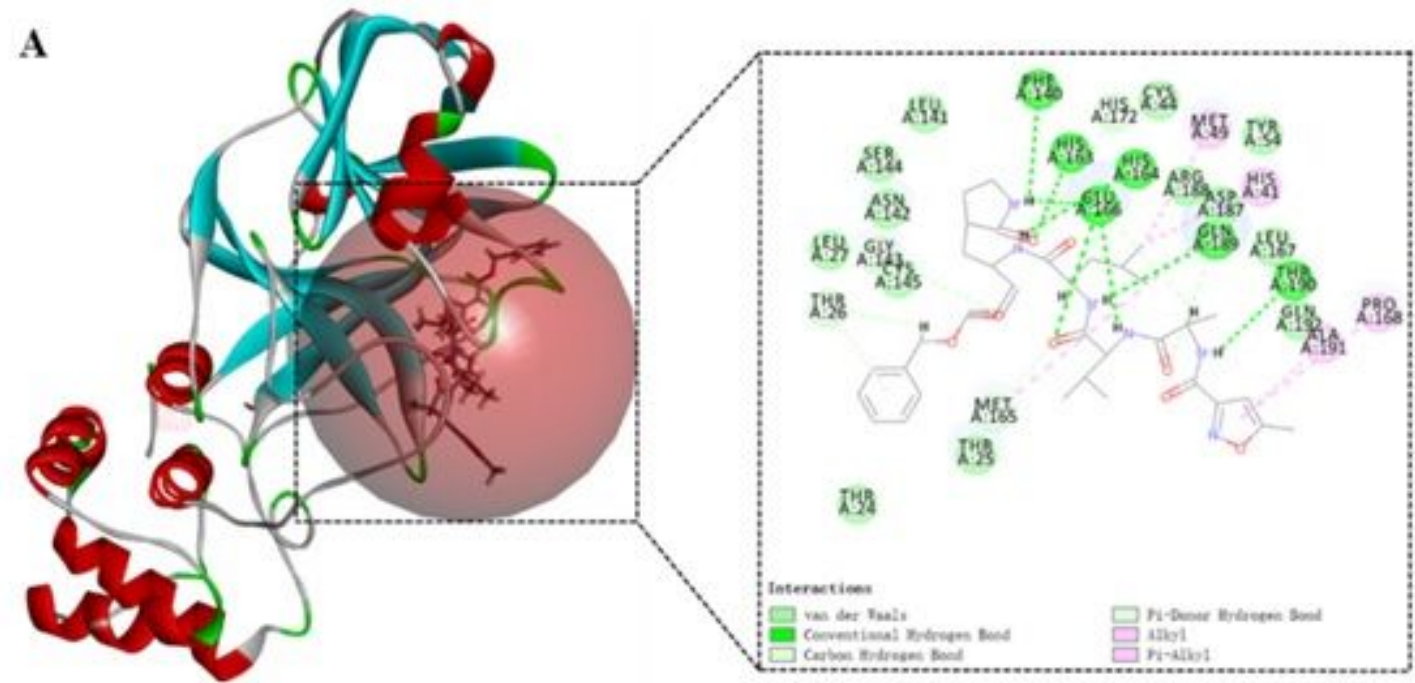

B

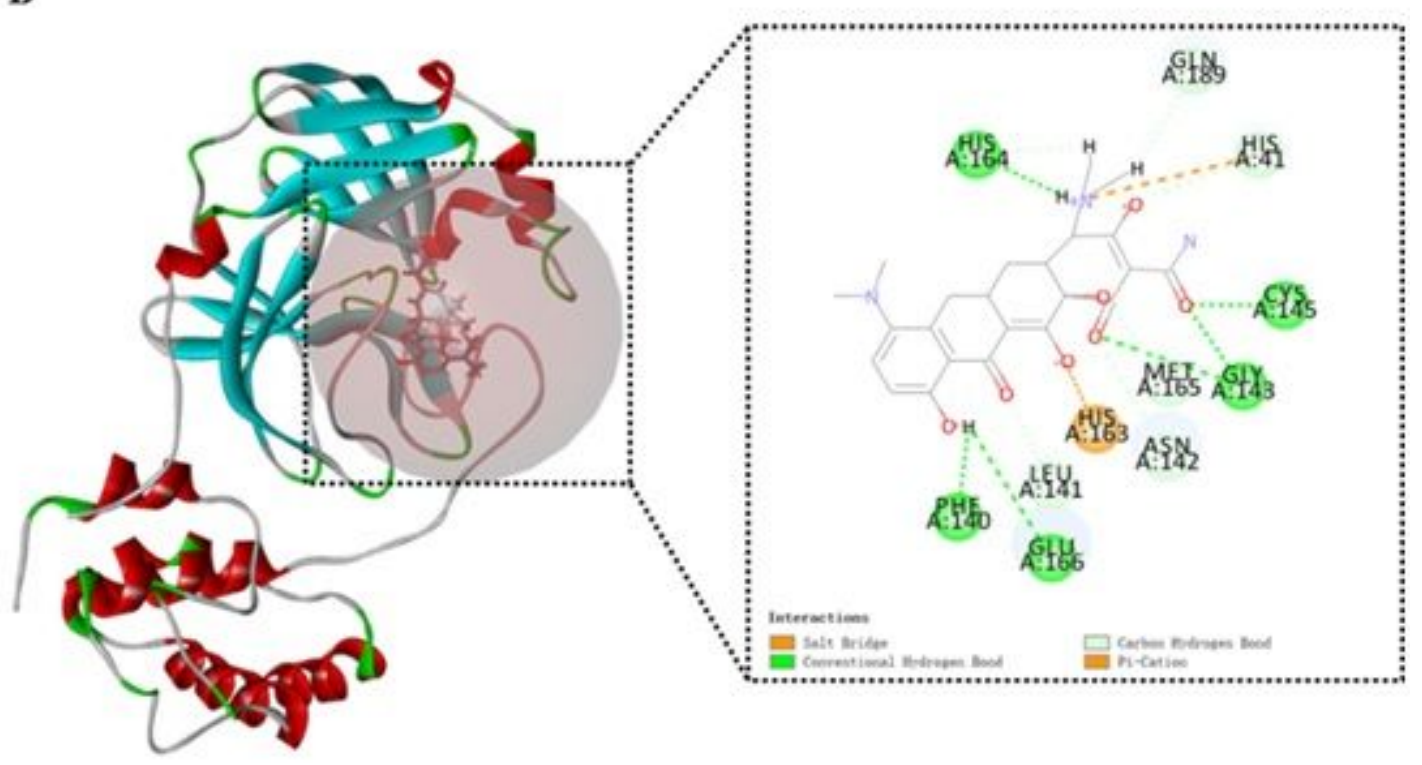

Figure 2

(A) Diagram of the interactions between N3 and Mpro; (B) Diagram of the interactions between minocycline and Mpro.The key amino acid residue is shown in sphere;Salt-bridge is shown in orange dashed line; Conventional hydrogen bond is shown in green dashed line; Carbon hydrogen bond is shown in light blue dashed line; Pi-cation is shown in bright-orange dashed line. 


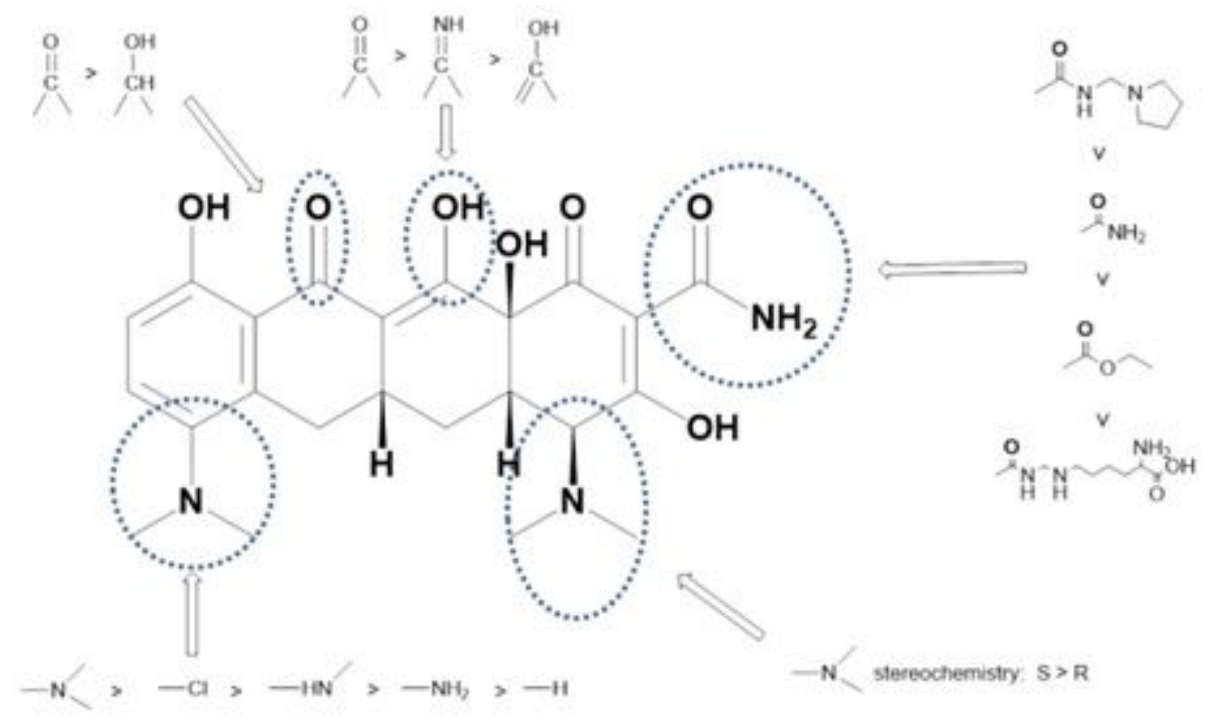

Figure 3

Primary structure-affinity relationship illustration ofminocycline targeting onMpro.

\section{Supplementary Files}

This is a list of supplementary files associated with this preprint. Click to download.

- SupportinginformationTableS2.docx

- SupportinginformationTableS2.docx

- SupportinginformationTableS1.doc

- SupportinginformationTableS1.doc 